\title{
Evaluación del desarrollo ruminal de corderos lanados y corderos media sangre Santa Inés faenados a diferentes pesos
}

\author{
Martinez, E. ${ }^{1}$; Yáñez, E.A. ${ }^{1}$; F.Quintana, C. ${ }^{2}$; Fernández, J.A. ${ }^{3}$ \\ ${ }^{1}$ Cátedra Nutrición y Alimentación, Fac.Cs.Veterinarias (FCV), Univ.Nac.Nordeste \\ (UNNE), Cabral 2139, Corrientes, Argentina. ${ }^{2}$ Cát.Histol. y Embriol. FCV, UNNE. \\ ${ }^{3}$ Cát.Nutr.Anim. Fac.Cs.Agrarias UNNE. E-mail: e.valeria.martinez@gmail.com
}

\begin{abstract}
Resumen
Martinez, E.; Yáñez, E.A.; F.Quintana, C.; Fernández, J.A.: Evaluación del desarrollo ruminal de corderos lanados y corderos media sangre Santa Inés faenados a diferentes pesos. Rev. Vet. 30: 2, $X X-X X$, 2019. En los últimos años se acentuaron los estudios del crecimiento y desarrollo de determinados órganos de los animales y sus implicancias en la productividad futura de los mismos. El objetivo del presente trabajo fue evaluar el desarrollo ruminal de corderos lanados y media sangre Santa Inés en diferentes pesos de faena. El ensayo fue realizado en el Departamento Monte Caseros, Provincia de Corrientes, Argentina. Se utilizó un lote de ovejas del biotipo lanado doble propósito de base Ideal. Para el servicio, la mitad de las ovejas lanadas fueron cruzadas con machos Santa Inés y la otra mitad, con reproductores Ideal, que fueron sincronizadas. El ensayo se realizó con los corderos lanados y media sangre (hembras lanadas $\mathrm{x}$ machos deslanados). El planteo experimental utilizado fue un diseño factorial por biotipo, sexo y peso de faena. Fueron sacrificados cinco animales por biotipo (lanados y media sangre), sexo (machos y hembras) y por cada peso de faena: 10, 17 y $24 \mathrm{~kg}$ de peso vivo promedio (en total 60 animales, 30 machos y 30 hembras). Las muestras fueron fijadas y posteriormente sometidas al procesamiento histológico de rutina. Los análisis morfométricos se realizaron utilizando un sistema analizador de imágenes. Se determinaron los espesores de la mucosa, epitelio y túnica muscular del rumen, así como la longitud y amplitud de las papilas, obteniéndose los promedios de las medidas. Los datos fueron sometidos a un análisis estadístico multivariado, realizando correlaciones entre las variables, análisis de componentes principales y análisis de la varianza. Las variables más influyentes en el proceso de desarrollo ruminal fueron: largo y ancho de papila, espesor del epitelio y grosor de la pared muscular. Los mayores tamaños de las mismas se observaron en los animales faenados a los $24 \mathrm{~kg}$ de peso vivo. Surge que el biotipo media sangre afecta el desarrollo de las variables, más en las hembras que en los machos.
\end{abstract}

Palabras clave: ovino, crecimiento, biotipo, desarrollo ruminal, sexo, peso.

Recibido: junio 2019 / Aceptado: julio 2019

\begin{abstract}
Martinez, E.; Yáñez, E.A.; F.Quintana, C.; Fernández, J.A.: Evaluation of ruminal development of woolly lambs and half blood Santa Inés lambs. Rev. Vet. 30: 2, XX-XX, 2019. In recent years, studies on the growth and development of certain animal organs and their implications for their future productivity have been emphasized. The objective of the present work was to evaluate the ruminal development of woolly lambs and half-
\end{abstract}


blood Santa Inés lambs at different slaughter weights. The trial was conducted in Monte Caseros Department, Corrientes Province, Argentina. A batch of sheep of dual-purpose woolly biotype (Ideal base), was used. For the service, half of the woolly sheeps were crossed with Santa Inés males, and the other half with Ideal breeding animals, which were synchronized. The test was carried out with the woolly lambs and half-blood Santa Inés lambs (woolly females x males Santa Inés). The experimental approach used was a factorial design by biotype, sex and slaughter weight. Five animals were slaughtered by biotype (wool and half-blood), sex (males and females) and by each slaughter weight: 10, 17 and $24 \mathrm{~kg}$ (30 males and 30 females). The samples were fixed and then subjected to routine histological processing. The morphometric analysis were performed using an image analyzer system. The thickness of the mucous membrane, epithelium and muscle tunic of the rumen were determined, as well as the length and amplitude of the papillae, obtaining the averages of the measurements. The data were subjected to a multivariate statistical analysis, making correlations between the variables, analysis of principal components and analysis of the variance. The most influential variables in the process of ruminal development were length and width of papillae, thickness of the epithelium and thickness of the muscular wall. The largest sizes of them were observed in the animals slaughtered at $24 \mathrm{~kg}$ of live weight. It appears that the half blood biotype affects the development of the variables, more in females than in males.

Key words: sheep, growth, biotype, ruminal development, sex, weight.

\section{INTRODUCCIÓN}

En los últimos años se acentuaron los estudios del crecimiento y desarrollo de determinados órganos de los animales de cría, así como sus implicancias en la futura productividad de los mismos ${ }^{3}$. El conocimiento de las características de tales procesos permite influir sobre ellos para lograr una mayor y más eficiente producción.

Si bien el crecimiento de los animales generalmente se asocia al aumento del peso en función del tiempo, este último constituye un indicador impreciso que no necesariamente refleja los cambios a nivel de tejidos, componentes corporales y metabolismo ${ }^{19}$.

La producción de rumiantes, en especial de bovinos, ovinos, y caprinos, implica la gran posibilidad de manipular su desarrollo para alcanzar alta productividad con mayor eficiencia y bajos costos, aprovechando la totalidad del potencial de los animales.

El sistema gastrointestinal de los rumiantes sufre cambios apreciables entre el nacimiento y el momento en que el rumen se torna funcional. En particular, los estudios realizados en ovinos demuestran que dichos cambios son promovidos por diferentes factores, tales como cantidad y calidad de alimentos, los cuales determinan el desarrollo y la diferenciación de los compartimentos del aparato digestivo.

Las sustancias producidas a través del metabolismo de los alimentos en el rumen, por ejemplo los ácidos grasos volátiles, estimulan el desarrollo de las diferentes estructuras del animal, tales como los epitelios y la pared muscular ${ }^{17}$. Dichos cambios promueven el aumento del peso y tamaño de los animales. Por lo tanto, entender el control del crecimiento y desarrollo ruminal, es esencial para establecer regímenes alimentarios que contribuyan a estos procesos de manera eficiente ${ }^{4}$. 
A pesar de los adelantos tecnológicos alcanzados en bioquímica e ingeniería genética, aún se busca un sustento en el significado biológico de las modificaciones del tamaño y forma de los animales, asociados a la funcionalidad de los órganos. Para ello es necesario vincular investigaciones anatómicas, fisiológicas y nutricionales, ya que varios fenómenos biológicos están involucrados ${ }^{14}$.

La incorporación de la morfología en los criterios de evaluación y selección de planes de mejoría a nivel mundial están basados en el crecimiento y desarrollo de los animales en función de la alimentación que reciben, las condiciones climáticas ${ }^{20}$, el estado sanitario ${ }^{11,18}$ y las características inherentes al biotipo y la raza.

En la zona productora ovina del nordeste argentino, el clima es una transición entre templado y subtropical, con inviernos generalmente benignos, aunque no exentos de heladas. Esto influye directamente en las características cuali y cuantitativas (digestibilidad, concentración de nutrientes, disponibilidad de materia seca, altura, densidad) de las praderas, que a su vez son las variables que influencian los parámetros de comportamiento ingestivo ${ }^{6,10}$, afectando el crecimiento y desarrollo de los animales. Las razas de pelo tienen un papel destacado en los cruzamientos con razas laneras, debido a su alta capacidad de adaptación a diferentes medios y sus ventajas reproductivas ${ }^{13}$.

El objetivo del presente trabajo fue evaluar el desarrollo ruminal de corderos lanados y media sangre Santa Inés con diferentes pesos de faena.

\section{MATERIAL Y MÉTODOS}

El ensayo fue realizado en un establecimiento del Departamento Monte Caseros, Provincia de Corrientes, Argentina, ubicado en el km 361,5 de la RN 14. Se utilizó un lote de ovejas del biotipo lanado doble propósito de base Ideal. Para el servicio, la mitad de las ovejas lanadas fueron cruzadas con machos Santa Inés y la otra mitad, con reproductores Ideal. Los vientres fueron sincronizados en sus ciclos estrales para obtener nacimientos de corderos homogéneos en cuanto a su edad.

El servicio fue de 45 días (marzo/abril). Se realizó en esta época por considerarla la más adecuada para las razas ovinas lanadas, predominantes en la región. El ensayo se realizó con corderos lanados y media sangre (hembras lanadas x machos deslanados), clasificados según el sexo e identificados en forma individual con caravanas numeradas y de diferentes colores.

El control sanitario de la majada incluyó vacunaciones contra enfermedades clostridiales y pediluvios con formol o sulfato de cobre para evitar enfermedades podales. También se realizaron análisis coproparasitológicos, con desparasitaciones estratégicas de acuerdo a la carga parasitaria detectada.

En la base forrajera disponible, además del pastizal natural típico de la región fitogeográfica "monte de ñandubay “, se contó con 25 ha de pastizal natural mejorado con leguminosas (lotus -Lotus corniculatus- y trébol blanco -Trifolium repens-). Se realizó una suplementación con concentrados en la época crítica de disponibilidad forrajera. Se suplementó con mezcla mineral todo el año.

Se efectuó un manejo racional del pastoreo, utilizando un sistema de rotación de potreros, basado en la disponibilidad de forraje y en el manejo preventivo de la parasitosis. La totalidad de los animales fue manejada bajo las mismas condiciones, ocupando simultáneamente los mismos potreros. Por lo tanto, todos recibieron la misma dieta.

El diseño experimental utilizado fue un factorial por biotipo, sexo y peso de faena. Para la evaluación del rumen fueron sacrificados cinco animales por biotipo (lanados y 
media sangre), sexo (machos y hembras) y por cada peso de faena: 10, 17 y $24 \mathrm{~kg}$ de peso vivo (PV) promedio (60 animales en total, 30 machos y 30 hembras), considerándose cada uno de los animales como una repetición.

El sacrificio se realizó previa insensibilización por aturdimiento eléctrico, siguiendo las directrices del código sanitario para animales terrestres de la Organización Mundial de Sanidad Animal ${ }^{12}$, seguido de sangría por sección de carótidas y yugulares.

Antes del sacrificio, los animales fueron sometidos a un ayuno de 12 h. Después de retirado el contenido del tubo gastrointestinal, fueron obtenidas muestras de pared ruminal para la evaluación tisular por microscopía. Las muestras fueron fijadas en solución de Bouin y posteriormente sometidas al procesamiento histológico de rutina.

Los estudios morfométricos se realizaron utilizando un sistema analizador de imágenes (Software Image Pro Plus, versión 4.5 Media Cybernetics, Inc.). De cada animal faenado se tomaron 3 muestras de diferentes sitios del órgano. De cada muestra se realizaron entre 5 a 10 fotografías de cortes histológicos.

Se determinaron los espesores de la mucosa, del epitelio y de la túnica muscular del rumen, como así también la longitud y amplitud de las papilas (las papilas medidas fueron aquéllas de tamaño medio, cuyo ancho se valoró en su porción media) (Figuras 1 y 2). Luego se calcularon los promedios de todas las medidas obtenidas para cada variable.

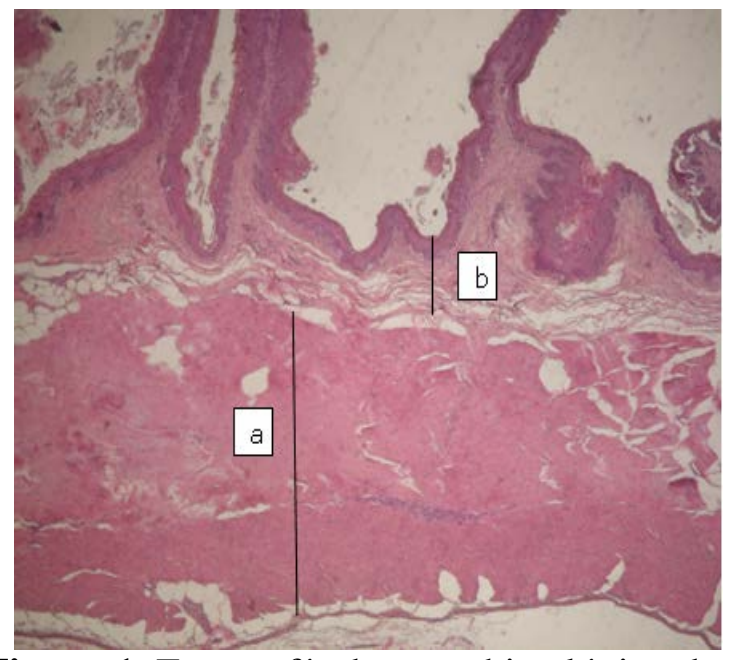

Figura 1. Fotografía de corte histológico de rumen. a: espesor de pared muscular, b: espesor de la mucosa 


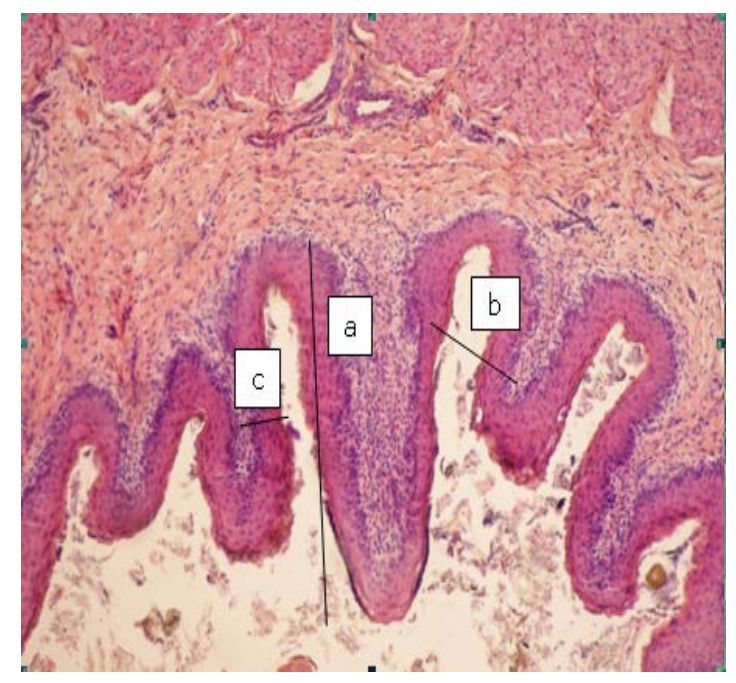

Figura 2. Fotografía de corte histológico de rumen.

a: largo de papila, b: ancho de papila, c: espesor del epitelio

Los datos obtenidos fueron procesados estadísticamente con el programa Infostat $2013^{7}$, mediante un análisis estadístico multivariado, realizando correlaciones entre las variables, estudio de componentes principales y análisis de la varianza entre las medias utilizando como variables de clasificación: el biotipo, el sexo y el peso de faena.

\section{RESULTADOS Y DISCUSIÓN}

En la Tabla 1 se muestran los promedios de medidas de variables por biotipo y peso de faena, sin distinción de sexo.

Tabla 1. Promedios de medidas de variables (en micras) por biotipo y peso de faena, sin distinción de sexo.

\begin{tabular}{lccccc}
\hline & \multicolumn{5}{c}{ variables ruminales } \\
\cline { 2 - 6 } & L.pap & A.pap & E.epit & E.musc & E.muc \\
\hline media sangre 10 kg & 530,15 & 228,45 & 78,04 & 895,17 & 195,93 \\
lanados 10 kg & 623,94 & 250,99 & 91,36 & 962,38 & 196,26 \\
media sangre 17 kg & 615,12 & 202,99 & 54,56 & 1063,61 & 255,68 \\
lanados 17 kg & 850,30 & 285,80 & 98,40 & 827,85 & 204,44 \\
media sangre 24 kg & 773,66 & 296,66 & 100,61 & 1054,77 & 284,83 \\
lanados 24 kg & 771,51 & 257,51 & 97,30 & 969,33 & 236,19 \\
\hline
\end{tabular}

L.pap: largo de papila; A.pap: ancho de papila; E.epit: espesor del epitelio; E.musc: espesor de la pared muscular; E.muc: espesor de la mucosa.

En el análisis de correlaciones entre las variables, utilizando como criterios de clasificación el biotipo y el sexo, se observó alta correlación positiva entre las variables ancho de papila y espesor del epitelio $(0,86)$ y alta correlación negativa entre largo de papila y ancho de papila $(-0,69)$, espesor de la pared muscular $(-0,79)$ y espesor de la mucosa $(-0,80)$. 
Considerando como criterios de clasificación al biotipo y peso de faena, el análisis de correlación reveló alta correlación positiva entre las variables ancho y largo de papila $(0,80)$ y entre ancho de papila y espesor del epitelio $(0,92)$. Esto indica que las variables que mantienen entre sí alta correlación son el ancho de papila y el espesor del epitelio en ambos criterios de clasificación.

La alta correlación negativa sugiere que a medida que hubo aumentos en estas variables, hubo menor desarrollo de las demás estructuras como la pared muscular y el espesor de la mucosa. Esto se evidenció solamente en la división por sexo, no por peso de faena. Posiblemente, a medida que los animales crecen y se empieza a desarrollar la mucosa y pared muscular, las diferencias con las demás variables no son tan marcadas.

Los estudios del normal desarrollo del rumen afirman que se requiere el establecimiento de una población microbiana viable y la formación de ácidos grasos volátiles para que se estimule el desarrollo de las diferentes capas del mismo, sobre todo la correspondiente a la mucosa ${ }^{16,21}$.

Con respecto al análisis de componentes principales, utilizando como criterios de clasificación el biotipo y el sexo (Figura 3), se observó que los dos primeros componentes explicaron el 98\% de la variabilidad de los datos. En el primer componente (61\%), las variables que más aportaron fueron la longitud de la papila, el grosor de la pared muscular y el espesor de la mucosa (estas dos últimas, en sentido inverso). En el segundo componente, la variable de mayor influencia fue el espesor del epitelio.

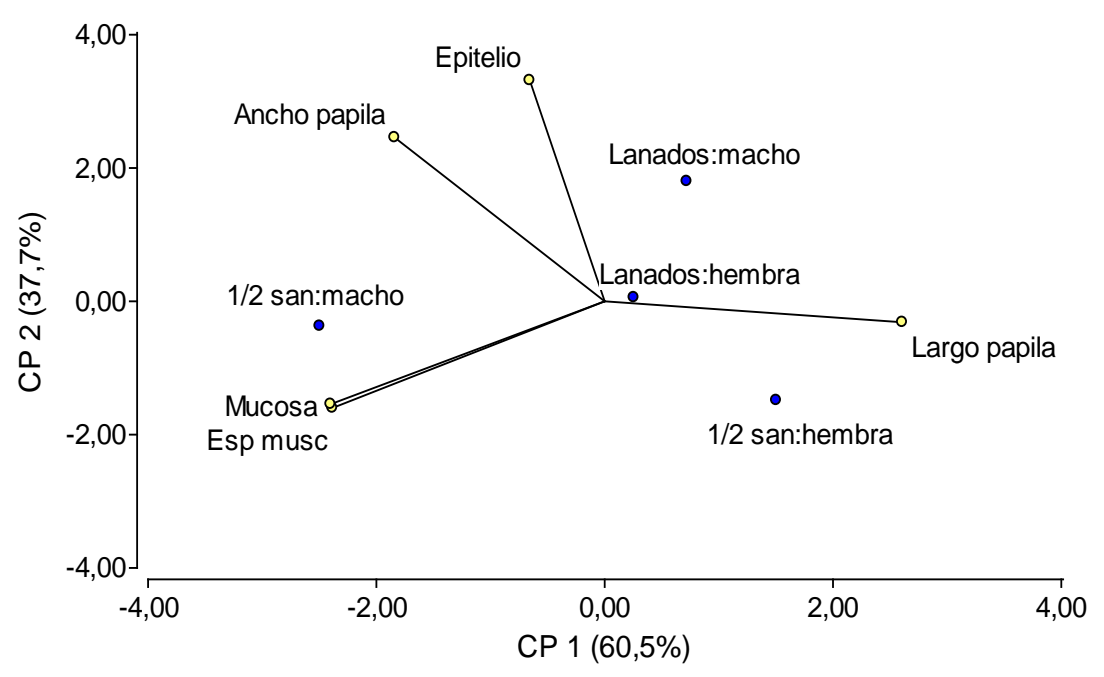

Figura 3. Distribución de las variables y su relación con el biotipo y sexo en los componentes principales (CP). Esp musc: espesor de la pared muscular; Mucosa: espesor de la mucosa; Ancho papila, Epitelio: espesor del epitelio; Largo papila). 1/2 san: biotipo media sangre Santa Inés; Lanados: biotipo lanados.

La Figura 3 demuestra que los animales media sangre fueron los que se encontraron más cerca de las variables que explicaban mayor versatilidad en el componente principal 1 (CP 1), ya sea de manera positiva o negativa. Esto sugiere que tales animales fueron los que presentaron mayor variación en esas estructuras y que el biotipo media sangre afectó al desarrollo de las mismas. 
En la formación de los componentes principales según biotipo y peso de faena (Figura 4), se observó que dos de ellos explicaron el 91\% de la variabilidad. Entre los mismos, el componente de mayor variabilidad (54\%), fue explicado por la longitud, el ancho de la papila y el espesor del epitelio.

Los animales lanados de 17 y $24 \mathrm{~kg}$, así como los ejemplares media sangre de 24 $\mathrm{kg}$ de peso de faena, se encontraron cercanos a las variables de mayor influencia en el CP 1 (ancho y largo de papila, así como espesor del epitelio). Sin embargo, los animales media sangre de $17 \mathrm{~kg}$ se encontraron alejados de estas últimas y en sentido contrario (negativo). En el componente principal 2 (CP 2), los animales de $10 \mathrm{~kg}$ se encontraron alejados del sentido de las variables, al igual que los ejemplares media sangre de $17 \mathrm{~kg}$.

A su vez, entre los animales de $10 \mathrm{~kg}$ de peso de faena, los ejemplares media sangre se encontraron más cerca de las variables "largo y ancho de papila" mientras que los lanados estuvieron más cercanos a la variable “espesor de la mucosa” (Figura 4).

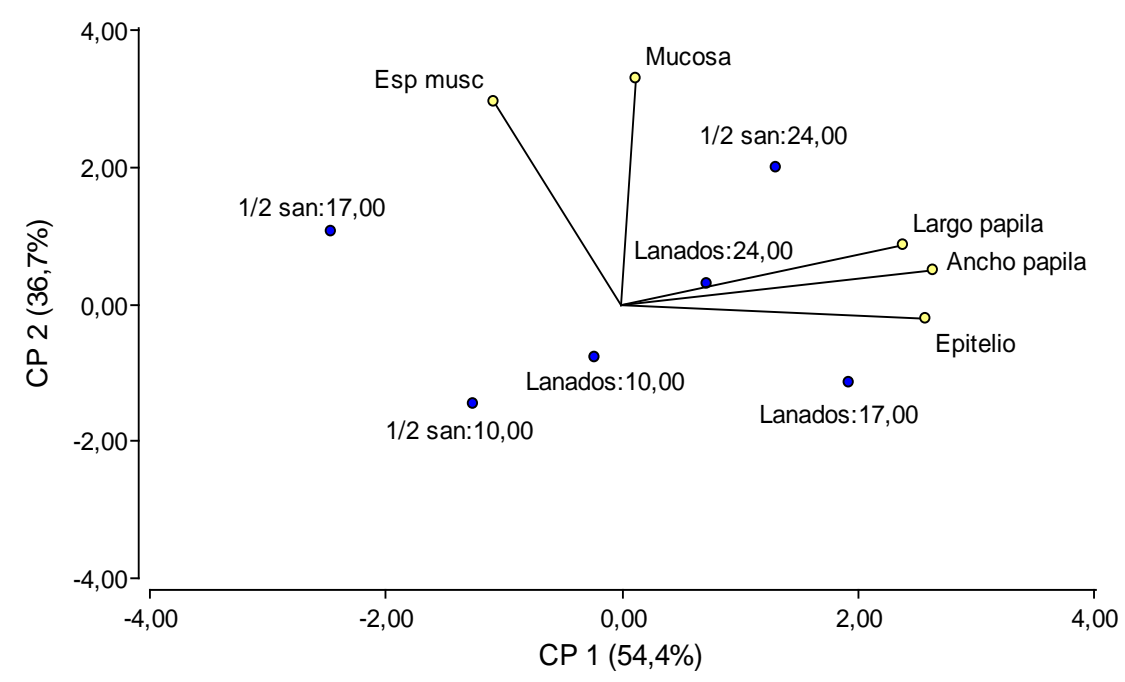

Figura 4. Distribución de las variables y su relación con el biotipo y el peso de faena en los componentes principales (CP). Esp musc: espesor de la pared muscular; Mucosa: espesor de la mucosa; Ancho papila; Epitelio: espesor del epitelio; Largo papila). 1/2 san: biotipo media sangre

Santa Inés; Lanados: biotipo lanado. 10, 17 y 24 kg: pesos de faena.

Esta característica posiblemente se deba a que los primeros empezaron a consumir alimentos sólidos antes que los segundos. Cuanto más alto fue el peso de los corderos, mayores fueron los cambios en dichas estructuras, los cuales fueron influenciados por el biotipo de los animales.

El peso vivo, la edad y el tipo de dieta influyeron en el inicio del desarrollo de las estructuras del rumen. A menores pesos de faena, la alimentación fue exclusivamente láctea, pero al aumentar el peso los corderos comenzaron a consumir alimentos groseros. Tal característica coincide con la verificada en otras investigaciones, donde el número de células y el total de la masa de pre-estómagos respondieron más al tipo de dieta que al consumo, incrementándose con los alimentos de contenido fibroso ${ }^{15}$.

Otros estudios confirmaron que en el rumen el establecimiento de la fermentación microbiana comienza entre las 2 y 4 semanas de edad, como resultado de la iniciación 
del consumo de alimentos sólidos ${ }^{2}$. En el presente trabajo, los animales de $10 \mathrm{~kg}$ de peso tenían aproximadamente 30 días de edad y no presentaban muchos cambios en las estructuras de desarrollo ruminal.

Los animales de 17 y $24 \mathrm{~kg}$ de peso vivo (entre 90 y 120 días de vida), revelaron cambios más evidentes, en coincidencia con los resultados de otros investigadores que compararon el desarrollo de corderos alimentados con leche y alimento iniciador (a los 56 días de edad no hubo diferencias significativas entre ambos tratamientos). A los 84 días, los corderos que consumieron ad libitum el alimento iniciador, tuvieron mayor desarrollo de la capa muscular y del epitelio ruminal. A su vez, lograron mayores ganancias diarias de peso que aquéllos que consumieron solamente leche ${ }^{21}$.

Otros trabajos describieron mayores ritmos de crecimiento en el peri-destete, pero los resultados confirmaron que a edades muy tempranas, incluso con dietas lácteas, se pueden detectar procesos incipientes de fermentación microbiana en el rumen ${ }^{5}$. Por su parte, otros investigadores realizaron trabajos con razas específicas y constataron que en corderos Santa Inês el desarrollo del rumen se completa al alcanzar los $15 \mathrm{~kg}$ de peso, lo cual implica que el cordero ya es apto para el cambio de dieta ${ }^{8}$. En el presente trabajo, en corderos media sangre Santa Inés, se observaron mayores evidencias de desarrollo ruminal, entre los 17 y $24 \mathrm{~kg}$ de peso vivo.

Las diferencias en las medias de las variables entre distintos pesos de faena coincidieron con lo arrojado por el análisis de la varianza $(\mathrm{p}<0,05)$ (Tabla 2). El hecho de que el espesor de la pared muscular no mostrara variación evidente en los diferentes pesos de faena, pudo deberse a que la ingesta de alimentos fibrosos no fue suficiente para estimular el desarrollo de esta estructura.

Tabla 2. Análisis de la variancia

\begin{tabular}{cc}
\hline $\mathrm{fv}$ & $\mathrm{p}$ \\
\hline animal & 0,1328 \\
trat faena & $0,0037^{\mathrm{a}}$ \\
sexo & 0,3830
\end{tabular}

fv: factor de variación; p: p-valor (grado de significancia); trat faena: peso vivo a la faena; ${ }^{\text {a }}$ diferencia significativa.

Ello coincide con resultados de estudios donde se observó que el estímulo físico ruminal resulta en un incremento de la musculatura del rumen pero no del desarrollo papilar. Al contrario, incrementos de concentrados en la dieta, no implicaron aumento de la musculatura, pero sí desarrollo de las papilas ${ }^{3}$.

Se afirma que el cambio entre la energía entregada por la leche versus el alimento sólido, causa sustanciales cambios en el desarrollo de la musculatura ruminal ${ }^{1}$. Otros estudios aseveran que la estimulación física generada por el alimento, se traduce en un importante incremento en el peso del rumen y desarrollo de la musculatura ${ }^{3}$.

Sin embargo ella no promueve el desarrollo papilar ${ }^{3,9}$, por lo cual, para un normal y progresivo desarrollo del epitelio y de las papilas ruminales, se debe establecer una viable fermentación. Ello indica que se requiere la presencia de ácidos grasos volátiles (AGV) como productos de una alimentación más concentrada en el lumen ruminal, para promover el normal desarrollo papilar ${ }^{3}$. 
En este trabajo, si bien las variables que expresaron mayores diferencias de peso fueron la longitud y el ancho de las papilas, posiblemente no se diferenciaron significativamente de otras estructuras del órgano por no existir una fermentación ruminal neta. Por consiguiente, las concentraciones de los AGV no resultaron suficientes para estimular el desarrollo de tales estructuras.

No obstante, los alimentos fibrosos son los que producen aumento de la proporción de ácido acético en el rumen, lo que genera cambios en el epitelio ruminal. La ingesta de estos alimentos, aunque en menores cantidades, podría ser la causa del cambio en el desarrollo del epitelio ruminal, en coincidencia con lo aseverado por investigadores que administraron infusiones con AGV conteniendo alta proporción de acetato, el cual demostró poseer efecto estimulatorio en el desarrollo del epitelio ruminal ${ }^{21}$.

Sin embargo, infusiones de butirato y propionato en el rumen, resultaron en un marcado desarrollo de las papilas ruminales ${ }^{3,16,21}$. Esto también podría explicar la poca variación del espesor de la mucosa ruminal registrada en el presente trabajo. Otros estudios revelaron que en relación al peso corporal, el crecimiento de la mucosa ruminal fue estimulado solamente en los periodos de ingestión de alimentos sólidos. El crecimiento de la mucosa ruminal responde al consumo de alimentos concentrados y es más marcado después de los 37 días de edad ${ }^{9}$.

Como conclusión, surge que las variables más influyentes en el proceso de desarrollo ruminal fueron el largo y ancho de las papilas, el espesor del epitelio y el grosor de la pared muscular. Los mayores tamaños se observaron en los animales faenados a los $24 \mathrm{~kg}$ de peso vivo. El biotipo media sangre afectó al desarrollo de las variables, más en las hembras que en los machos.

Agradecimientos. A la empresa Gesgan Asesores SRL, por ceder los animales utilizados en el ensayo. Al Establecimiento Don Donato, por permitir el uso del lugar, estructuras y materiales. Al Sr. Arnoldo Dri, por la colaboración brindada durante el trabajo.

\section{REFERENCIAS}

1. Baldwin RL, Jesse BW. 1992. Developmental changes in glucose and butyrate metabolism by isolated sheep ruminal cells. J Nutr 122: 1149-1152.

2. Baldwin RL. 1999. The proliferative actions of insulin, insulin-like growth factor-I, epidermal cells in vitro. Small Rum Res 32: 261-268.

3. Baldwin RL. 2000. Sheep gastrointestinal development in response to different dietary treatments. Small Rum Res 35: 39-47.

4. Baldwin RL, McLeod KR, Klotz JL, Heitmann RN. 2004. Rumen development, intestinal growth and hepatic metabolism in the pre- and postweaning ruminant. $J$ Dairy Sci 87 (E.Suppl.): E55-E65.

5. Belanche A, Fuente G, Yañez DR, Calleja L, Balcells J. 2007. Desarrollo anatómico y microbiológico del rumen: efecto de la edad y tipo de dieta. Rev ITEA 28: 276278.

6. Chilibroste P, Soca P, Bentancur O, Mattiauda DA. 2010. Estudio de la conducta en pastoreo de vacas Holando de alta producción. Rev Agrociencia (Uruguay) 14: 3, 101-106.

7. Di Rienzo J et al. 2013. Infostat versión 2013. Grupo InfoStat, FCA, UNC, URL: http://www.infostat.com.ar 
8. Freitas MA, Ungerfeld R. 2016. Destete artificial en ovinos: respuesta de estrés y bienestar animal. Rev Mex Ciencias Pecuarias 7: 361-375.

9. Hamada T, Maeda S, Kameoka K. 1976. Factors, influencing growth of rumen, liver and others organs in kids weaned from milk replacers to solid foods. J Dairy Sci 59: 1110-1118.

10. Hodgson J. 1986. Grazing behaviour and herbage intake. XIX Symp of UK British Grassland Soc, www.britishgrassland.com/page/contact-bgs-team, p. 51-64.

11. Martínez GS et al. 2010. Tecnologías para mejorar la producción ovina en México. Rev Fuente 5: 41-51.

12. Organización Mundial de Sanidad Animal. 2007. Código sanitario para los animales terrestres, $16^{\circ}$ ed., Cap 7.5. www.oie.int/doc/ged/D6435.PDF

13. Partida JA, Braña VD, Jiménez SH, Ríos FG, Buendía RG. 2013. Producción de carne ovina. Anales Centr Nac Investig Fisiol Anim, Ajuchitlán. México. www.anetif.org/files/pages/0000000034/20.pdf

14. Pereda ME. 2003. Crecimiento y desarrollo de las especies pecuarias. Rev Agrofaz 3: $213-220$.

15. Sainz RD, Bentley BE. 1997. Visceral organ mass and cellularity in growthrestricted and refed beef steers. J Anim Sci 75: 1229-1236.

16. Sander EG, Warner HN, Harrison HN, Loosli JK. 1959. The stimulatory effect of sodium butyrate and sodium propionate in the development of rumen mucosa in the young calf. J Dairy Sci 42: 1600-1605.

17. Sakata T, Tamate H. 1978. Rumen epithelial cell proliferation accelerated by rapid increase in intraruminal butyrate. J Dairy Sci 61: 1109-1113.

18. Searle AC. 1999. Prevalencia y factores de riesgo de algunas alteraciones crónicas de la glándula mamaria en ovinos de 20 pequeñas explotaciones de la Provincia de Valdivia, Chile. Univ Austr de Chile, Fac Cs Vet www.veterinariaudec.cl/

19. Soto N, Mericq V. 2005. Restricción del crecimiento fetal e insulinorresistencia. Nuevos hallazgos y revisión de la literatura. Rev Méd Chile 133: 97-104.

20. Stringini A. 2015. Morfometría geométrica: estudio de asimetría en cráneos ovinos procedentes de tres diferentes áreas geográficas catalanas. Univ. Lérida, Grado de Ciencia y Salud Animal. Repositori.udl.cat/bitstream/handle/10459.1/48977/strin gini.pdf

21. Tamate H, McGilliard AD, Jacobson NL, Getty R. 1962. Effect of various dietaries on the anatomical development of the stomach in the calf. $J$ Dairy Sci 45: 408-420. 